\title{
EFFECTS OF WHOLE-BODY VIBRATION ON THE PERCEIVED DURATION OF A VISUAL STIMULUS PRESENTATION
}

\author{
Kazuma Ishimatsu*, Nobuyuki Shibata, Setsuo Maeda \\ National Institute of Occupational Safety and Health, Japan (JNIOSH)
}

\section{Introduction}

Timing of intervals in the seconds-to-minutes range, which is involved in foraging and decision making, is essential for representing the immediate external environment ${ }^{1}$. This is evident in many daily activities such as safely crossing a busy street, driving a vehicle, preparing a meal, and so on. For example, while driving down or crossing a busy street, speed and time estimates are continuously required. There is much evidence to show that subjective judgment of duration (i.e., time estimation) can be lengthened or shortened by non-temporal factors. Whole-body vibration (WBV) could be one of those factors affecting subjective judgment of duration ${ }^{2}$. The current study investigated whether time estimation abilities (i.e., verbal estimations) could be affected by exposure to WBV.

\section{Methods}

Participants. Eleven university students (9 females, 2 males) were paid participants. All participants gave written informed consent before taking part in this study, which was approved by the research ethics committee of the JNIOSH.

Apparatus and stimuli. A color AV-tachistoscope (IS-703, IWATSU ISEC Co. Ltd.) controlled timing of the events and generated the stimuli. The stimuli were presented on a 22-inch color monitor. A vibrator (ATS-II V, Akashi Corporation) was used to generate sinusoidal vertical vibration at a magnitude of $1.0 \mathrm{~ms}^{-2}$ r.m.s. (unweighted acceleration). The frequency of the vibration used in this experiment was $5 \mathrm{~Hz}$ and $16 \mathrm{~Hz}$.

Procedure. Participants were tested individually. The participant sat on a seat of the vibrator, and was given instructions. As this was a prospective verbal estimation paradigm, participants were instructed in advance that their task would be to estimate in seconds how long a visual stimulus was presented in each trial. Four 10-s time estimation trials were administered, as well as four 25-s trials, four 45-s trials, and four 60 -s trials. These intervals were presented in a random sequence of 16 trials. Followed by a practice block, each participant performed the task during each of the following three conditions: a baseline block of trials without vibration $(0 \mathrm{~Hz})$; a block with $5 \mathrm{~Hz}$ vibration; and a block with $16 \mathrm{~Hz}$ vibration. The order of performing the experimental blocks was counterbalanced across participants. Within each experimental block, the order of the presentation duration of target was randomly determined for each participant separately. No feedback was given regarding the accuracy of verbal estimates in all experimental blocks. At the end of each experimental block, each participant reported a rating of discomfort from the WBV (from 1, not uncomfortable to 5, very uncomfortable) as well as a rating of distraction by the WBV (from 1, not distracted to 5, very distracted).

\section{Results}

Four scores were derived from the verbal time estimation task ${ }^{3}$ : Mean time estimates, absolute error values (ABS), a coefficient of variance (CV), and a duration judgment ratio. Mean time estimates represented the raw scores calculated for each time 
duration interval. The ABS provided an overall measure of accuracy so that if a particular participant tended to error in the direction of both over- and underestimation, the average error would not tend toward zero. The CV allowed us to evaluate how consistent participants were in their verbal estimates of the same target interval. Duration judgment ratios provided an index of accuracy regardless of the size of the standard interval.

Mean score. Analyses of the raw scores revealed a significant main effect for time interval, $F(3,30)=116.30, M S E=82.33, p<.01$. The main effect of block and the Block $x$ Time interval interaction did not reach statistical significance, $p s>.20$. Consistent with the time perception literature, which indicates that individuals typically underestimate time, the verbal estimates represented an underestimation of time relative to the actual intervals.

$A B S$ score. The ANOVA of the ABS scores revealed a significant main effect for time interval, $F(3,30)=12.90, M S E=72.48, p<.01$. The main effect of block and the Block $\mathrm{x}$ Time interval interaction did not reach statistical significance, $p \mathrm{~s}>.15$. These indicate that participants' estimates tended to increasingly deviate from true clock time as the interval to be estimated lengthened.

$C V$ score. The ANOVA of the CV scores revealed no significant main effects of time interval and block, or Block x Time interval interaction, $p s>.20$.

Duration judgment ratio score. The ANOVA on the ratio score revealed no significant main effects of time interval and block, or Block x Time interval interaction, $p s>.20$. These findings indicate that the ratio of estimated time to clock time remained stable across time intervals for all three conditions.

Discomfort and Distraction. The discomfort was worst in the $5 \mathrm{~Hz}$ block $(M=3.2)$, the second worst in the $16 \mathrm{~Hz}$ block $(M=2.4)$, and least in the $0 \mathrm{~Hz}$ block $(M=1.0), x^{2}(2)=$ $17.15, \mathrm{p}<.01$. Ratings of the distraction in the vibration blocks [i.e., $5 \mathrm{~Hz}(M=3.5)$ or 16 $\mathrm{Hz}(M=2.9)]$ were significantly worse than that of the $0 \mathrm{~Hz}$ block $(M=1.1), x^{2}(2)=$ $17.68, \mathrm{p}<.01$.

\section{Discussion}

The purpose of this study was to investigate whether time estimation abilities (i.e., verbal estimations) could be deteriorated during exposure to WBV. The present study revealed that the WBV used in this experiment could not interfere with verbal estimation performances although participants reported that verbal estimations were distracted by the WBV. This indicated the discrepancy between the verbal estimations and subjective ratings. The distraction in the vibration blocks was worse than that of the baseline block without vibration. Additionally, effects of WBV on discomfort ratings were found. The discomfort in the $5 \mathrm{~Hz}$ block was worse than that of the $16 \mathrm{~Hz}$ block, suggesting that the effect of WBV could be frequency-dependent. Taken together, in conclusion, time estimation abilities could not be deteriorated during exposure to whole-body vibration.

\section{References}

1. Block, R.A., Zakay, D., and Hancock, P.A. (1998). Human aging and duration judgments: A metaanalytic review. Psychology and Aging, 13, 584-596.

2. Ishimatsu, K., Shibata, N., and Maeda, S. (2009). Time perception during exposure to whole-body vibration. Proceedings of 44th United Kingdom Conference on Human Responses to Vibration, 179-187. 3. Rueda, A.D., and Schmitter-Edgecombe, M. (2009). Time estimation abilities in mild cognitive impairment and Alzheimer's disease. Neuropsychology, 23, 178-188. 